\title{
Essai d'entretien de cellules épithéliales de reins de bovins par passages répétés et modification du milieu nutritif
}

\author{
Note préliminaire \\ Mme Josette MONNIER-CAMBON
}

\begin{abstract}
RESUME
II a été possible d'effectuer avec succès 27 passages de cellules épithéliales de rein de bovin durant une période de quatre mois.

La vilesse de croissance des cellules s'est améliorée du 1er passage au $5^{\mathrm{E}}$ et une modification du milieu de culture a permis de la maintenir jusqu'au $20^{\circ}$. Du $20^{\mathrm{e}}$ passage au $27 \mathrm{e}$, la vitalité des cellules dans ce même milieu est allée en diminuant. Au $28^{\circ}$ passage, le tapis cellulaire n'a pu arriver à recouvrir que 5 pour cent de la boîte de Roux.

Cette lignée cellulaire au $11^{\mathrm{e}}$ passage ef $13^{\mathrm{e}}$ passage s'est montrée utilisable pour la croissance et le titrage du virus bovipestıque. La souche Kabete « $O$ » aussi bien que la souche « B » Dakar donnent des lésions identiques à celles observées sur des cellules de 1 re explantalion ef surtout un titre égal.

Une étude des chromosomes réalısée sur des cellules du $10^{\mathrm{e}}$ passage a permis de constater que le nombre modal n'étalt pas modifié et que la morphologie ne

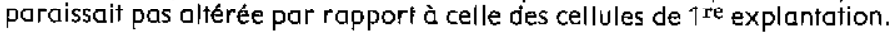

L'application de la technique de culture cellulaire pour la croissance ou l'isolement d'un virus tant dans le domaine de la recherche que dans celui du diagnostic, devient de plus en plus fréquente et il est évident que le fait de pouvoir bénéficier d'un matériel à jour fixe, est un avantage indiscutable. L'objet de cette éłude a donc été d'essayer d'obtenir une lignée cellulaire par passages répétés de cellules épithéliales en notant les conditions particulıères, s'il y a lieu, du développement.

\section{MATÉRIEL}

La suspension cellulaire de reins de foetus* bovin no 68 présentant une grande facilité de culture, le tapis cellulaire atteint 95 p. 100 en 30 h sur une boîte de Roux, est retenue pour être à l'origine de cet essai.

\section{TECHNIQUE}

10 Préparation des cellules de Ire explantation

Les reins d'un foetus bovin ( 7 mois environ) sont prélevés aseptiquement. La substance corticale est hachée finement à l'aide de scalpels, lavée trois fois dans une solution tamponnée salée (PBS)** ef mise au contact d'une solution de trypsıne Difco à 0,3 p. 100 en Hanks BSS***. Après une prétrypsination de $20 \mathrm{mn}$ à la température du laboratoire, la trypsination proprement dite est conduite à $+4^{\circ}$ pendant $5 \mathrm{~h}$. La trypsine est ensuite séparée par centrifugation.

* Les embryons n'élant utilisés qu'âgés d'au moins trois mois et plus, le terme « fœetus » est préféré. Conf. BLIN et FOURNIER (1).

** PBS $=$ phosphate buffer solution (Dulbecco).

*** BSS = balanced salt solution. 
Le culot cellulaire est lavé et mis en culture à raison de 1 volume pour 250 dans du Hanks LAYE* (pH 7,2) auquel on ajoute 10 p. 100 de sérum de veau (importé de France) ainsi que des antibiotiques, pénicilline $150 \mathrm{U}$. l., streptomycine $150 \gamma$ et mycostatine $100 \mathrm{U}$. I. par ml. Après répartition, les cultures sont incubées à $37^{\circ}$.

Les renouvellements ultérieurs de milieu sont effectués tous les 2 ou 3 jours à l'aide du même milieu, mais la proportion de sérum est ramenée à 5 p. 100 de sérum de bouf (importé de France) au 1 er changement et 2 p. 100 pour les suivants. Le $\mathrm{pH}$ est porté de 7,2 à 7,4 par adjonction d'une solution de bicarbonate. Cetfe méthode a été exposée plus en détail dans un précédent article (2).

\section{$2^{\circ}$ Obtention de sub-culture}

Huit jours après la mise en culture, le tapis d'un flacon de $250 \mathrm{ml}$ est lavé trois fois avec une solution de trypsine Difco à 0,3 p. 100 en solution salée, sans calcium ni magnésium (CMFS). Au dernier lavage, le flacon Incliné est placé à l'étuve à 370 pendant quelques $m n$ de telle sorte que la quantité infime de trypsine résıduelle Imprègne le tapis cellulaire, et, pour éviter toute dessiccation, la trypsine est passée sur le tapis cellulaire par inclinaisons répétées du flacon. Dès que les cellules sont décollées, elles sont remises en suspension dans une partie aliquote du milieu où elles seront cultivées ( 2 fois le volume initial). Pour ce premier passage, la composition est la même que pour la mise en culture de cellules de première explantation. If est très important de pipetter et repipetter plusieurs fois afin de diviser le plus possible les amas cellulaires : leur croissance sera d'autant plus rapıde que les cellules auront été plus isolées.

\section{RÉSULTATS}

\section{Croissance cellulaire.}

Cinq jours après, la crosssance a été telle qu'il est possible de refaire un passage et jusqu'au cinquième passage, ce délai de cinq jours sera observé. A partir de celui-ci, trois jours seulement sont nécessaires pour obtenir un tapis complet.

\footnotetext{
* LAYE = hydrolysat de lactalbumine ef extrait de levure.
}

En même temps, on note que les cellules deviennent plus rondes et que leur croissance est améliorée en modifiant les conditions du milieu.

\section{a) Adjonction de bicarbonate.}

Au jour J, trypsination d'un flacon de $250 \mathrm{ml}$ (5e passage) et mise en culture dans 2 flacons. Le premier, dans les conditions habituelles, le deuxième après adjonction de bicarbonate afin d'obtenir un $\mathrm{pH}$ aux environs de 7,4-7,5.

Au jour $J+1$, le tapis est le sulvant*:

flacon sans bicarbonate ...... 30 p. 100

flacon avec bicarbonate ....... 95 p. 100

Au jour J +2 :

flacon sans bicarbonate ...... 40 p. 100

fiacon avec bicarbonate ...... 100 p. 100

\section{b) Augmentation de la teneur du milieu en} sérum.

Les cellules obtenves par trypsination d'un autre flacon du $5^{e}$ passage sont d'abord portées à la dilution habituelle de 1 volume pour 2 volumes. La moitié de la suspension obtenue est distribuée, l'autre moitié est encore diluée à volume égal et mise en culture en quantité convenable. La proportion de sérum dans ce dernier milıeu est augmentée à 12 p. 100 et 48 h après, les deux cultures ont atteint également 100 p. 100 de développement.

Au $7 \mathrm{e}$ passage, quelques temps de croissance sont relevés :

- A 13 h, trypsination du 6e passage et mise en culture dans un flacon de $250 \mathrm{ml}$ et de tubes Leighton.

- A 19 h 30 , le tapis est le suivant : 45 p. 100 dans les tubes, 60 p. 100 dans le flacon.

- A 7 h le lendemain :

80 p. 100 dans les tubes, 95 p. 100 dans le flacon.

On peut donc constater une croissance extrêmement rapide dans les premières heures, et, contrairement à ce qui se passe habituellement à un tel niveau de passage, on n'observe pas une majorité de fibroblastes. L'ensemble est bien formé de cellules épithéliales (voir photos 1, 2 et 3 ).

* Le pourcentage est évalué de façon approximative en effectuant une moyenne des résultats donnés par plusieurs lectures au microscope. 


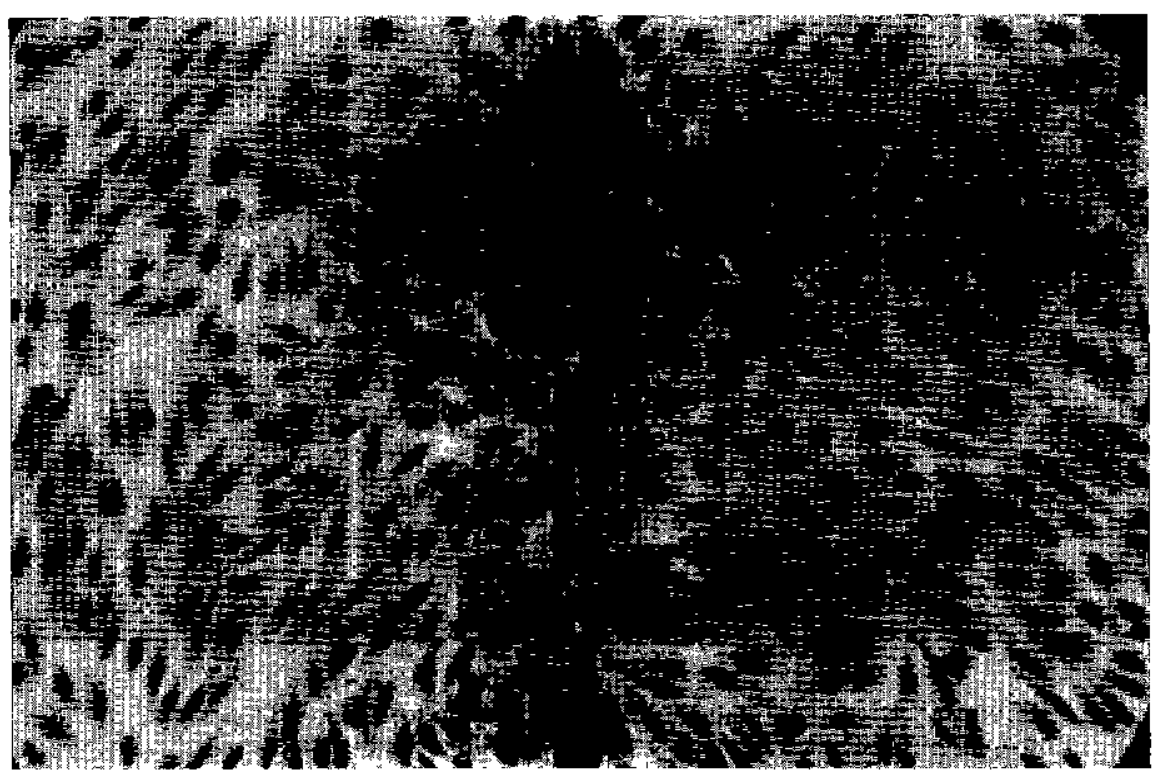

Fig. 1. - Culture de cellules épithéliales de reın de foetus bovin au 6 e jour, 7e passage, $\times 160$.

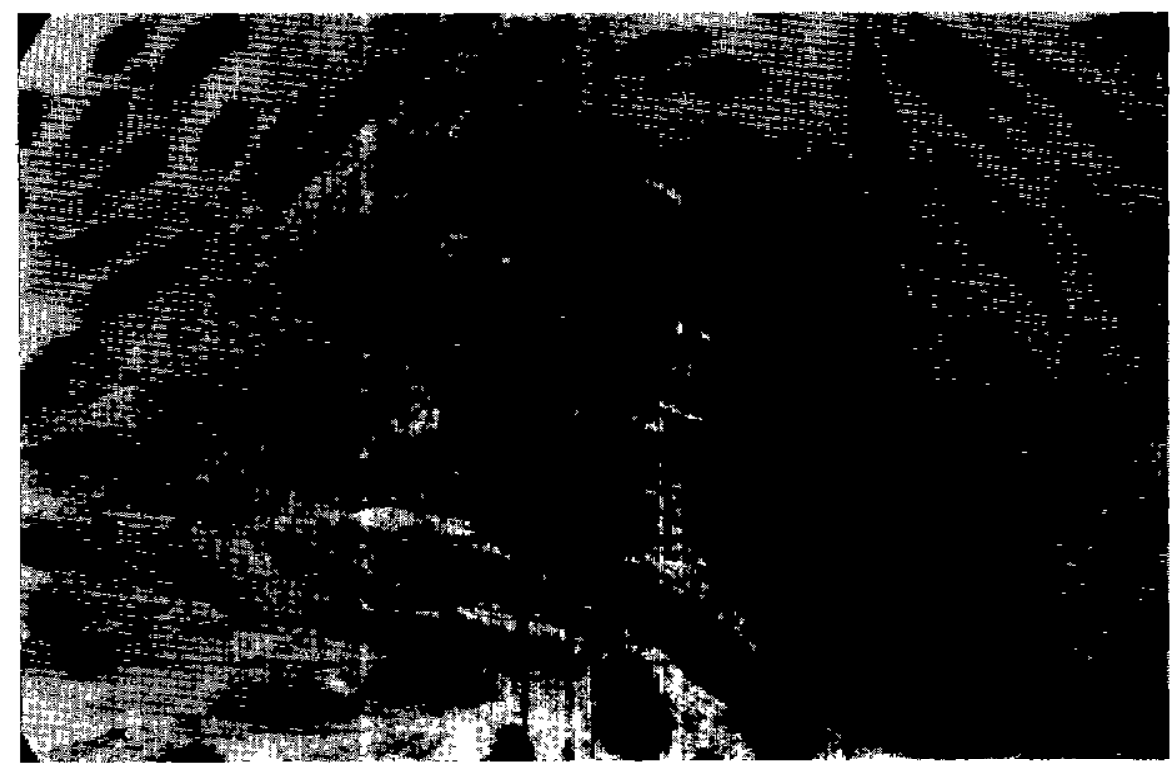

Fig. 2. - Cutture de cellules épithéliales de rein de foetus bovin au $3 \mathrm{e}$ jour, $7 e$ passage, $\times 400$. 


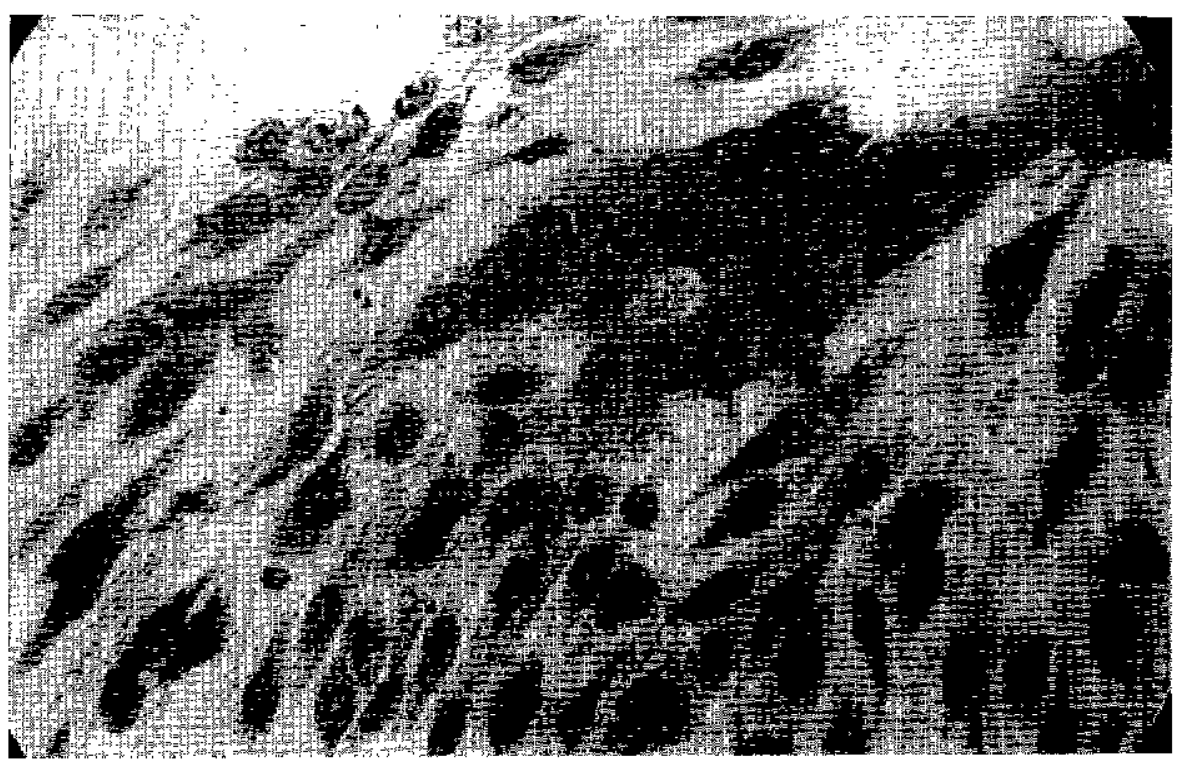

Fig. 3. - Culture de cellules épithéliales de rein de foetus bovin (10e passage) infectées par le virus bovipestique Kabele « $O » 4$ jour à 370 - Cellules multinucléées avec vacuale $-X 400$.

Malheureusement, les passages ne sont pas poursuivis au-delà du $28^{\mathrm{e}}$. Avant le $20^{\mathrm{e}}$ passage, la viłalité des cellules est telle qu'un 'flacon de $250 \mathrm{ml}$ permet ia mise en culture de 4 flacons de même volume. Mais depuis le 23 e passage, la faculté de reproduction diminue et une composition convenable du milieu, peut-être encore à modifier, n'a pu être étudiée par suite de circonstances indépendantes de notre volonté, les cellules fibroblastiques deviennent très abondantes.

Au moment où la vitesse de croissance des cellules était maxima, une étude est entreprise sur la valeur qualitative de ces cellules. Elle porte sur:

- la culture du virus bovipestique,

- le comportement génétique.

\section{Culture et titrage du virus bovipestique.}

Des titrages comparés de virus sont effectués en utilisant la souche bovipestıque « $B$ » Dakar.

- virus sur cellules de 1 re explantation.

- virus sur cellules du $11^{\text {e }}$ passage titré à la fois sur cellules de 1 re explantation et sur cellules du $13^{\mathrm{e}}$ passage. (Tableau).

\begin{tabular}{|c|c|c|}
\hline & $\begin{array}{l}\text { Cultivé sur cellules } \\
\text { lère explantation }\end{array}$ & $\begin{array}{l}\text { Cultivé sur cellules } \\
\text { 11ène passage }\end{array}$ \\
\hline Virus & \begin{tabular}{l|l} 
Titre $\left(\mathrm{DI}_{50} \mathrm{Ct}\right)$ sur \\
cell.ules lère \\
explentation
\end{tabular} & cellules lère $\int_{\text {explantation }} \int_{\text {passage }}$ cellules 13 ème \\
\hline "B" & $10^{-5,5}$ & $10^{-5,3}$ \\
\hline
\end{tabular}


La souche bovipestique Kabete « $O$ » cultivée sur cellules de 1 re explantation et sur cellules du $13^{\mathrm{e}}$ passage, donne dans les deux cas un titre de $10^{-5},^{2}$.

La lignée cellulaire obtenue parâ̂t avoir gardé vis-à-vis du virus bovipestique, son caractère réagissant, tout en ayant multiplié son facteur de croissance. S'agit-il d'une mutation ? II est difficile d'affirmer ou d'infirmer ce terme, tous les moyens d'étude ne pouvant être envisagés. L'étude des chromosomes entreprise au 10e passage va cependant pouvoir nous donner une idée sur le matériel génétique de cette celiule.

\section{Etude des chromosomes.}

La première expérience réalisée sur des cellules ayant à peine $24 \mathrm{~h}$ s'avère nulle. Aucun chromosome n'est sorti sauf sur les lames comportant des cellules normales et dont la croissance se situait entre $48 \mathrm{~h}$ et $3 \mathrm{j}$.

La deuxième expérience est réalisée sur des cellules de presque $3 \mathrm{j}$ et cette fois avec succès. A ce propos se pose le problème de la reproduction, pourtant si rapide dans les premières heures.

Au jour J, le $9^{e}$ passage est trypsiné et mis en culture notamment dans 10 tubes Leighton. Au jour $\mathrm{J}+2$ les milieux sont changés en diminuant la quantité totale ( $1 \mathrm{ml}$ au lieu de 2 ) et en augmentant la teneur en sérum du milieu (20 p. 100).

6 h après, 6 tubes reçoivent 3 gouttes d'extr ait embryonnaıre de poussin. Au jour $\mathrm{J}+3$, on introduit une goutte à 2 gouttes d'une solution de colchicine à $0,4 \mathrm{mg}$ p. 100 dans chaque tube (1). On laisse $4 \mathrm{~h}$ à l'éfuve à $37 \circ \mathrm{C}$. Ce produit, ainsi qu'il est connu, a pour effet de bloquer la mitose en métaphase. Au bout de ce temps, on vide le milieu et on ajoute par tube $1 \mathrm{ml}$ d'un mélange réchauffé à $37^{\circ} \mathrm{C}$ d'eau distillée, d'eau physiologique et de hyaluronidase. Les tubes sont agités légèrement, laissés $35 \mathrm{mn}$ à la température du laboratoire. Il se produit alors un éclatement des cellules. C'est ensuite la fixation au Cormoy (alcool absolu, chloroforme, acide acétique), puis l'hydrolyse dans un bain-marie à $60^{\circ}$ en présence d'acide chlorhydrique normal. La coloration se fait au bleu de toluidine, le

(1) Technique pratiquée au laboratoire d'hygiène de la Faculté de Médecine (Prof. agrégé BAYLET et Mme GRATTEPANCHE que nous remercions de leurs conseils). montage au baume du Canada sirupeux. Parallèlement, une série de lamelles de 1re explantation est examinée.

Immédiatement après séchage, les préparations sont observées au microscope. On repère les plaques sur lesquelles les chromosomes sont nets et séparés afin d'obtenir des photographies suffisamment démonstratives. Après agrandissement de celles-ci, les chromosomes sont découpés un à un, groupés par paire et par ordre décroissant de taille.

Une comparaison de plaques de chromosomes résultant des cellules de 1 re explantation et du 10 e passage montre que, malgré l'entretien en milieu artificiel et 10 passages, ni le nombre, ni la morphologie des chromosomes ne semblent altérés. Les figures « 4 » et « 5 » montrent respectivement les chromosomes en métaphase de cellules épithéliales de rein de foetus bovin de 1 re explantation et du $10^{\mathrm{e}}$ passage.

\section{CONCLUSION}

II a été possible d'effectuer avec succès 27 passages de cellules épithéliales de rein de bovin durant une période de 4 mols.

La vitesse de croissance des cellules s'est améliorée du 1 er passage au $5^{\mathrm{e}}$ et une modification du milieu de culture a permis de la maintenir jusqu'au 20e. Du $20^{\circ}$ passage au $27 \mathrm{e}$, la vitalité des cellules dans ce même milieu est allée en diminuant. Le 28e passage n'a pu arriver qu'à 5 p. 100 de tapis.

Cette lignée cellulaire au 11 e passage et $13 \mathrm{e}$ passage s'est montrée utilisable pour la craissance ef le titrage du virus bovipestique. La souche Kabete « $O$ » aussi bien que la souche «B»Dakar donnent des lésions identiques à celles observées sur ces cellules de 1 re explantation et surtout un titre égal.

Une étude des chromosomes réalisée sur des cellules du $10^{\mathrm{e}}$ passage a permis de constater que le nombre modal n'était pas modifié ef que la morphologie ne paraissait pos altérée par rapport à celle des cellules de 1 re explantation.

Institut d'Elevage et de Médecine Vétérinare des Pays tropicaux Laborataire Notional

de l'Elevage et de Recherches Vétérinaires Dakar-Honn 


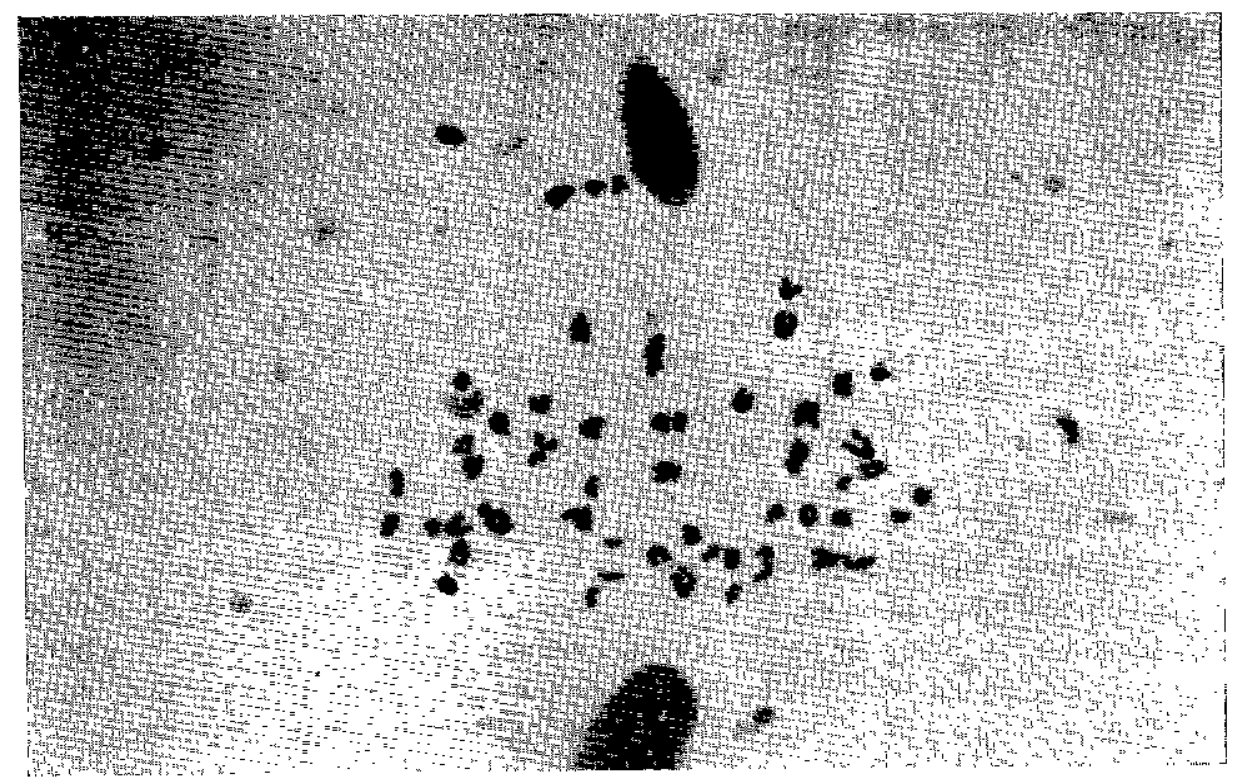

Fig. 4. - Chromosomes en métaphase de cellules épithéliales de rein de foetus bovin de 1 re explantation.

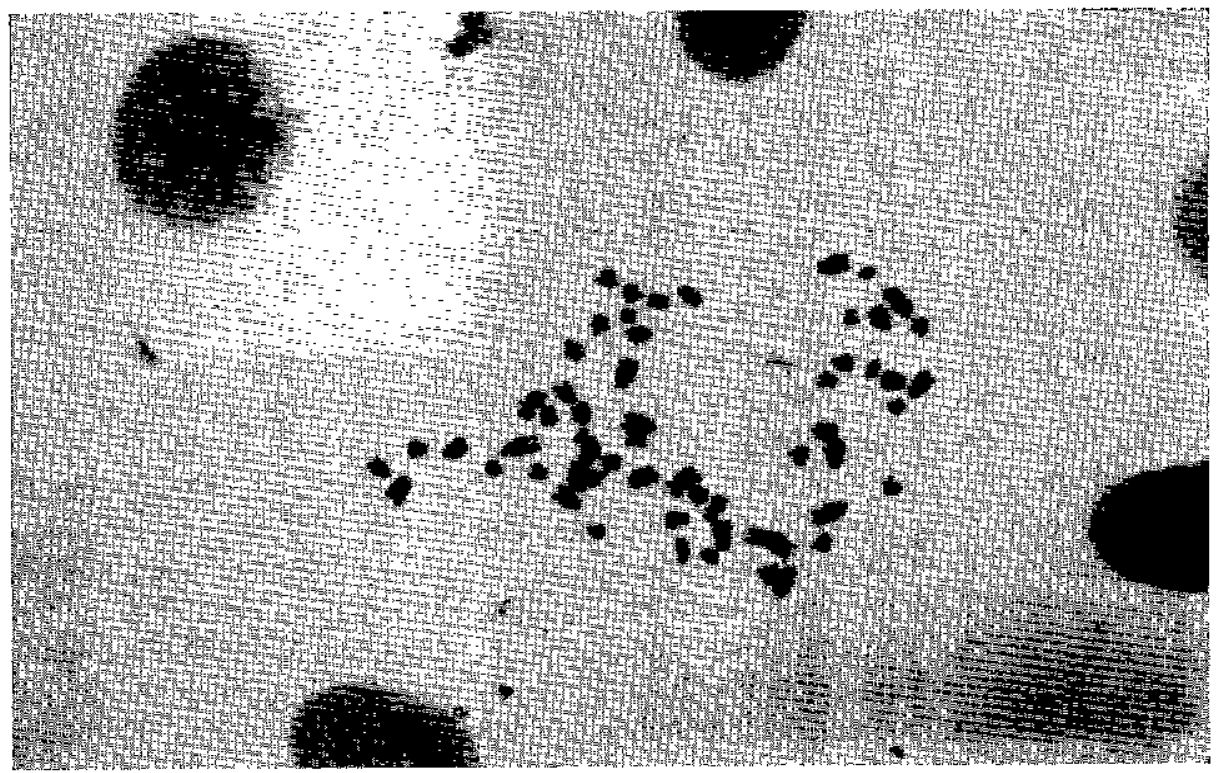

Fig. 5, - Chromosomes en métaphase de cellules épithéliales de ran de fotus bovin de $1^{\text {re }}$ explantation. $10^{\mathrm{e}}$ passage. 


\section{SUMMARY}

Attempt to maintain bovine kidney cells by repeated passages and madification of the nutritive medium. Preliminary note.

It was possible, to successfully carry out 27 successive passages of bovine kidney epithelial celis over a period of four months.

The rale af growth of the cells was improved from the 1 st to the 5 th passage and a madification of the culture medium enabled one to mantain it up to the 20 th. From the 20 th to 27 th passage, the vitality of the cells in this same medium progressively decreased. The 20 th passage produced 5 p. 100 of the covering growth only.

This strain, at the 11 th and 13 th passage, proved useful for the growth and assay of the rinderpest virus. The Kabele «O $O$ strain as well as the Dakar « $B$ » strain produced lesions identical to those observed on cells of primary culture : they gave also a same $\mathrm{TCI}_{50}$ titer.

The study of the chromosomes carried out on cells derived from the 10 th passage enabled one to ascertain that the modal number had not changed and that the marphology did not appear to be modified as compared to that of the cells of the 1 st explant.

\section{RESUMEN}

Ensayo de conservación de células epiteliales de riñon de bovinos mediante repetidos pasajes y modificación del medio nutritivo. Nota preliminar.

Se tuvo exito al efectuar 27 pasajes de células epiteliales de un riñon bovino durante cuatro meses.

La velocidad de crecımiento de las células está mejorada del 1 er pasaje al $5^{\circ}$, y una modificación del medio de cultivo permitió mantenerla hasta el $20^{\circ}$. Del $20^{\circ}$ pasaje al $27^{\circ}$, la viabilidad de las células en este mismo medio fué disminuyendo. Con el $28^{\circ}$ pasaje, la alfombra celular no pudo cubrir mâs que 5 por 100 de la superficie de la caja de Roux.

Esta linea celular con el $11^{\circ}$ y el $13^{\circ}$ pasaje se encontró utilizable para el crecimienta y la dosificación del virus bovipestico. La cepa Kabete « $\mathrm{O} »$ asi como la cepa «B » Dakar dan lesiones identicas a las observadas en celulas de primer cultivo y sobretodo un título semejante.

Un estudio de los cromosomas realizado en celulas del $10^{\circ}$ pasaje permitió comprobar que el número modal no eslaba modificado y que la morfologia no parecía alterada con respecto a la de las celulas de primera 'explantación.

\section{BIBLIOGRAPHIE}

1. BLIN (P. C.) \& FOURNIER (Cl.). - Diagnose de l'âge intra-maternel ef périodisation du développement dans l'espèce bovine. Economie ef Médecine animales $n^{0} 1,12-32$, 1963.

2. GlLBERT (Y.) et MONNIER (J.). - Adaptation d'une souche de virus bovipestique à la culture cellulaire. Premiers résultats. Rev. Elev. Méd. Vét. Pays trop., 1962, 15 (4), 311-20.

3. FERGUSSON (J.) \& WANSBROUGH (A.). Isolement et culture à long terme d'une lignée de cellules diploïdes de mammifères (Isolation and long term culture of diploid mammalian cell lines). Concer Res., 22, 556-562, 1962.
4. GRASSE (P. P.). - Traité de zoologie. T. XHI. 5. MEDDA (A.), MUNTONI (S.) et LODDO (B.). Caractéristiques de cellules rénales bovines propagées sérialement in vitro (caratteristiche di cellule renali bovine propagate serialmente in vitro. (Nota preventiva). Vét. ital. suppl. n० 6, 1962, 588-89.

6. TIJO (J. H.) \& PUCK (T.). - Génétique des cellules somatiques de mammifères II. Constifution chromosomique des cellules en culture de tissu (Genetics of somatic mammalian cells. II. Chromosomal constitution of cells in tissue culture). Journol of experimental Medicine, vol. 108, no 2, 259-268, 1958. 